\title{
Novel vortex structures in dipolar condensates
}

\author{
S. Yi and H. Pu \\ Department of Physics and Astronomy, and Rice Quantum Institute, \\ Rice University, Houston, TX 77251-1892, USA
}

(Dated: April 21, 2021)

\begin{abstract}
We investigate the properties of single vortices and of vortex lattice in a rotating dipolar condensate. We show that vortices in this system possess many novel features induced by the long-range anisotropic dipolar interaction between particles. For example, when the dipoles are polarized along the rotation axis, vortices may display a crater-like structure; when dipoles are polarized orthogonal to the rotation axis, vortex cores takes an elliptical shape and the vortex lattice no longer possesses hexagonal symmetry.
\end{abstract}

PACS numbers: 03.75.Lm, 03.75.Nt

Quantized vortices represent one of the fundamental topological structures in nature. Studies of quantized vortices were launched out of the efforts to understand the flow properties of superfluid ${ }^{4} \mathrm{He}$, but have now extended into a variety of systems including superconductors, superfluid ${ }^{3} \mathrm{He}$, quantum magnets, liquid crystals, nuclear matter and even cosmic strings [1]. The realization of weakly-interacting quantum gases of atomic vapors provides a new platform for the study of quantum vortices. Vortices in atomic Bose-Einstein condensates have been produced, perturbed and engineered by precise external fields, and can be studied theoretically in a quantitative manner using first-principles calculations [2]. Very recently, vortices were also observed in a quantum degenerate Fermi gas 3 .

So far, vortices have only been produced in condensates featuring short-range $s$-wave collisions. The creation of condensation in the transition metal ${ }^{52} \mathrm{Cr}$ offers a completely new system of quantum gases [4]. The ground state of ${ }^{52} \mathrm{Cr}$ has a large magnetic dipole moment of $\mu=6 \mu_{B}$, with $\mu_{B}$ being the Bohr magneton. Hence the magnetic dipolar interaction between atoms plays a crucial role in ${ }^{52} \mathrm{Cr}$ condensates. Previous theoretical studies of dipolar quantum gases have focused on the ground state properties of non-rotating systems [5]. The purpose of this paper is to investigate the properties of vortices in a rotating dipolar condensate. As we will show, due to the long-range and anisotropic nature of the dipolar interaction, single vortices and vortex lattices in such systems possess many unique features not present in condensates with only contact collisions.

Model - We consider a trapped condensate of $N{ }^{52} \mathrm{Cr}$ atoms whose dipole moments are polarized along certain direction. The two-body dipolar interaction potential is

$$
V_{d d}\left(\mathbf{r}, \mathbf{r}^{\prime}\right)=\frac{\mu_{0} \mu^{2}}{4 \pi} \frac{1-3 \cos ^{2} \theta}{\left|\mathbf{r}-\mathbf{r}^{\prime}\right|^{3}}
$$

where $\mu_{0}$ is the vacuum magnetic permeability, and $\theta$ is the angle between the dipole moment and the vector $\mathbf{r}-\mathbf{r}^{\prime}$. The trapping potential is assume to be harmonic with axial symmetry

$$
U(\mathbf{r})=\frac{1}{2} M \omega_{\perp}^{2}\left(x^{2}+y^{2}+\lambda^{2} z^{2}\right)
$$

with $\omega_{\perp}$ being the radial trap frequency and $\lambda=\omega_{z} / \omega_{\perp}$ the trap aspect ratio. For the sake of simplicity, we will focus on a pancake-shaped system with $\lambda \gg 1$ such that the condensate can be regarded as quasi twodimensional (2D) whose motion along the $z$-axis is frozen to the ground state of the axial harmonic oscillator. Therefore the condensate wave function can be decomposed as $\Psi(\mathbf{r}, t)=\psi(\boldsymbol{\rho}, t) \phi(z)$, where $\boldsymbol{\rho}=(x, y)$ and $\phi(z)=(\lambda / \pi)^{1 / 4} e^{-\lambda z^{2} / 2}$. Throughout this paper, we adopt a unit system where the units for length, frequency and energy are given by $a_{\perp}=\sqrt{\hbar / M \omega_{\perp}}, \omega_{\perp}$ and $\hbar \omega_{\perp}$, respectively. The reduction of the effective spatial dimensionality considerably reduces the computational intensity. Although intrinsic three-dimensional (3D) phenomena such as the bending and Kelvin mode excitation of vortex lines cannot be studied, the 2D model does capture many essential features of vortices at equilibrium, for example, the structure of the vortex core and that of vortex lattice.

We assume that the condensate is rotating along the symmetry axis $z$ with angular frequency $\Omega$. After integrating out the $z$-variable, we obtain the effective $2 \mathrm{D}$ Gross-Pitaevskii equation in the rotating frame as

$$
\begin{aligned}
i \frac{\partial \psi}{\partial t}= & {\left[-\frac{\nabla_{\perp}^{2}}{2}+\frac{\rho^{2}}{2}-\mu-\Omega L_{z}+g_{2 D}|\psi|^{2}\right.} \\
& \left.+\int d \boldsymbol{\rho}^{\prime}\left|\psi\left(\boldsymbol{\rho}^{\prime}\right)\right|^{2} V_{d d}^{2 D}\left(\boldsymbol{\rho}, \boldsymbol{\rho}^{\prime}\right)\right] \psi(\boldsymbol{\rho})
\end{aligned}
$$

where $\mu$ is the chemical potential, $L_{z}$ the $z$-component of the orbital angular momentum operator, $g_{2 D}=$ $2(2 \pi \lambda)^{1 / 2} N a / a_{\perp}$ the rescaled $2 \mathrm{D}$ collisional interaction strength with $a$ being the $s$-wave scattering length which can be tuned via Feshbach resonance [6]. The rescaled 2D dipolar interaction potential takes the form

$$
V_{d d}^{2 D}\left(\boldsymbol{\rho}, \boldsymbol{\rho}^{\prime}\right)=\int d z|\phi(z)|^{4} V_{d d}\left(\mathbf{r}, \mathbf{r}^{\prime}\right) .
$$

In the following, we will treat separately the cases where the dipoles are polarized along the axial and along the transverse direction, as they lead to very different phenomena. 


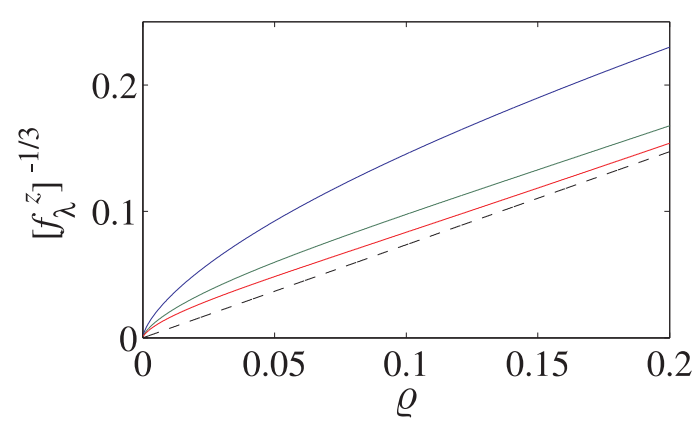

FIG. 1: (Color online). Solid lines: function $\left[f_{\lambda}^{z}(\varrho)\right]^{-1 / 3}$ for $\lambda=11,100$, and 400 in descending order. The dashed line represents the asymptote $(2 \pi)^{-1 / 6} \varrho$.

Axially polarized dipoles - For this case, we can find from Eq. (4) that

$$
V_{d d}^{2 D}\left(\boldsymbol{\rho}, \boldsymbol{\rho}^{\prime}\right)=\frac{N \mu_{0} \mu^{2}}{(2 \pi)^{3 / 2} a_{\perp}^{3} \hbar \omega_{\perp}} f_{\lambda}^{z}(\varrho),
$$

where $\varrho=\left|\boldsymbol{\rho}-\boldsymbol{\rho}^{\prime}\right|$ and

$$
\begin{aligned}
f_{\lambda}^{z}(\varrho)= & \frac{\lambda^{3 / 2} e^{\lambda \varrho^{2} / 4}}{6}\left[\lambda \varrho^{2} K_{0}\left(\frac{\lambda \varrho^{2}}{4}\right)+\left(2-\lambda \varrho^{2}\right)\right. \\
& \left.\times K_{1}\left(\frac{\lambda \varrho^{2}}{4}\right)\right]-\frac{\sqrt{\pi \lambda}}{\varrho^{2}} U\left(\frac{3}{2}, 0, \frac{\lambda \varrho^{2}}{2}\right),
\end{aligned}
$$

with $K_{n}(z)$ being the $n$ th-order modified Bessel function of the second kind and $U(a, b, z)$ being the confluent hypergeometric function. Fig. [1 shows the function $\left[f_{\lambda}^{z}(\varrho)\right]^{-1 / 3}$ for various values of the trap aspect ratio $\lambda$. It can be seen that, as expected, the effective dipolar interaction is repulsive and isotropic. Independent of $\lambda$, $f_{\lambda}^{z}(\varrho)$ asymptotically approaches $\sqrt{2 \pi} \varrho^{-3}$ at large $\varrho$. At the other limit, as $\varrho \rightarrow 0, f_{\lambda}^{z}(\varrho)$ diverges much slower than $\varrho^{-3}$, although the detailed behavior at small $\varrho$ depends on the value of $\lambda$. Note that for a true 2D system with dipoles located in the $x y$ plane and polarized along $z$ axis, one expects a dipolar interaction potential $\tilde{V}_{d d}^{2 D} \propto \varrho^{-3}$ as can be easily seen from Eq. (1). For the quasi-2D case considered here, the effective dipolar interaction potential deviates from $\tilde{V}_{d d}^{2 D}$ at small values of $\varrho$ in a fashion that makes the singularity of $V_{d d}^{2 D}$ at $\varrho=0$ integrable, a quite important property for numerical calculations. An alternative, and often more efficient, way to treat the dipolar terms in numerical calculations is to use the Fourier transform of $V_{d d}^{2 D}[7]$.

The ground state wave function can be obtained by evolving Eq. (3) in imaginary time. In the numerical results presented in the paper, we use $N=10^{5}$ and $\omega_{\perp}=$ $2 \pi \times 100 \mathrm{~Hz}$. First we want to study the structure of a single axial vortex at the center of the cloud. Due to the azimuthal symmetry, such a state can be written as $\psi(\boldsymbol{\rho})=\chi(\rho) e^{i \varphi}$ with $\varphi$ being the azimuthal angle and $\chi \mathrm{a}$ real function satisfying the following $1 \mathrm{D}$ radial equation:

$$
i \frac{\partial \chi}{\partial t}=\left[-\frac{1}{2 \rho} \frac{\partial}{\partial \rho}\left(\rho \frac{\partial}{\partial \rho}\right)+\frac{1}{2 \rho^{2}}+\frac{\rho^{2}}{2}+g_{2 D} \chi^{2}+\mathcal{D}(\rho)\right] \chi,
$$
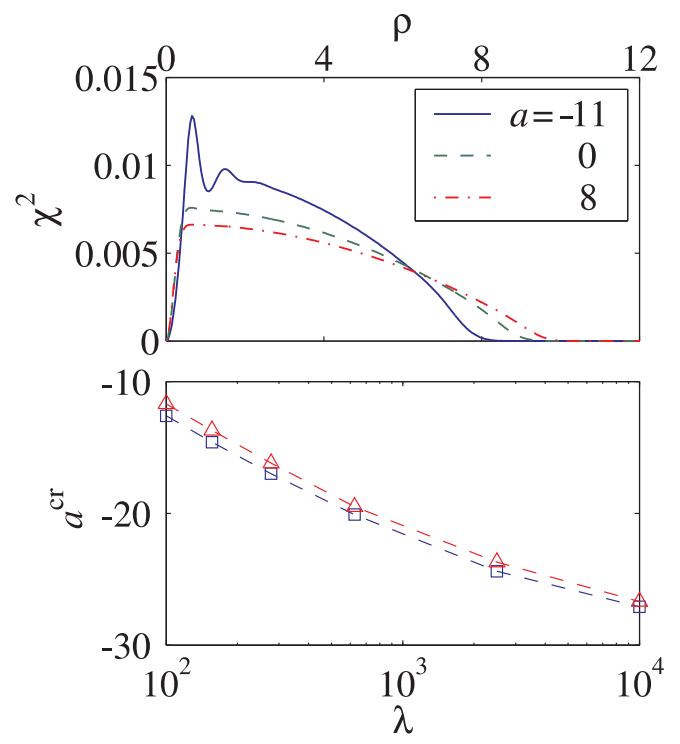

FIG. 2: (Color online). (a) Radial density profile of the single vortex state for $\lambda=100$ and various scattering lengths (in units of Bohr radius, $a_{B}$ ). (b) The $\lambda$ dependence of critical scattering length (in units of $a_{B}$ ). Here $\square$ and $\Delta$ denote, respectively, the non-vortex state and the axial vortex state.

where

$\mathcal{D}(\rho)=\int_{0}^{\infty} \rho^{\prime} d \rho^{\prime} \int_{0}^{2 \pi} d \varphi^{\prime} V_{d d}^{2 D}\left(\rho^{2}+\rho^{\prime 2}-2 \rho \rho^{\prime} \cos \varphi^{\prime}\right) \chi^{2}\left(\rho^{\prime}\right)$.

Figure 2(a) illustrates the radial density profile $\chi^{2}(\rho)$ of the single vortex state for different values of the scattering length. An interesting feature one can notice is that the density close to the vortex core oscillates as long as $a$ is not too large. Such oscillatory behavior is induced by the dipolar interaction and gives the vortex a crater-like shape. Similar density oscillations are also observed in numerical studies with other forms of nonlocal interaction potentials which are originally employed to model the inter-particle interactions in superfluid ${ }^{4} \mathrm{He}$ [8, 9]. Note that no oscillations are found in the ground state structures of a non-rotating dipolar condensate.

The density oscillations of the vortex state also affect its stability property. A condensate with sufficiently large and negative $a$ is unstable and tends to collapse [10]. The critical scattering length $a^{\text {cr }}$ as a function of the trap aspect ratio is shown in Fig. 2(b) for both the axial vortex state and the non-vortex state. Near $a^{\text {cr }}$, due to the dipole-induced density oscillation, the vortex state has a peak density exceeds that of the non-vortex state. Since collapse starts locally at the high density region, the vortex state tends to be less stable, having a critical scattering length smaller in magnitude by about $5 \sim 10 \%$ compared with the non-vortex state. This is in contrast with the non-dipolar condensate where the vortex states are shown to be more stable [11] as they have lower peak densities compared to the non-vortex states. We remark that the repulsive dipolar interaction in this 


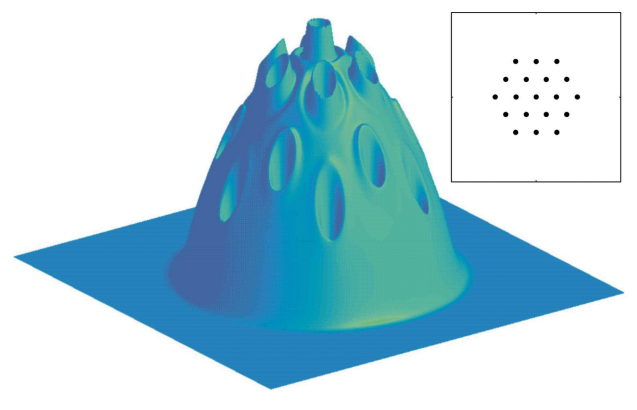

FIG. 3: (Color online). Density profile of a vortex lattice state for $\lambda=100, \Omega=0.4 \omega_{\perp}$, and $a=-10 a_{B}$. The inset shows the position of the vortices which form a triangular lattice with hexagonal symmetry.

case helps to stabilize a condensate with attractive scattering length: with all the other parameters being the same as in Fig. 2(b), a non-dipolar condensate would have a critical scattering length on the order of $-0.1 a_{B}$.

As we increase the rotation frequency, more vortices enter the condensate and form a regular lattice as a result of the repulsive vortex-vortex interaction. For a non-dipolar condensate, it is well known that the vortex lattice takes a triangular shape with hexagonal symmetry. Very recently, it is predicted by several authors that the vortex lattice of a dipolar condensate in the high-rotating quantum Hall limit may take different geometries [12, 13]. However, we find from our numerical calculations that the triangular lattice with hexagonal symmetry always has the lowest energy for rotating frequencies up to $\Omega=0.99 \omega_{\perp}$. Although computing power and numerical accuracy currently prevent us from taking higher values of $\Omega$, this suggests that the quantum Hall regime of a fast rotating dipolar condensate may need a more careful analysis. An example of the vortex lattice is shown in Fig. [3 where the crater structure is still present. To ensure that such a structure is not an artefact due to the reduction of the effective spatial dimension, we also performed full 3D calculations and found that the results are in complete agreement with the $2 \mathrm{D}$ calculation.

Transversely polarized dipoles - It is also of interest to study the vortex state with dipole moments polarized transversely. We assume that the dipoles are polarized by a transverse magnetic field co-rotating with the condensate about the $z$ axis [14]. Without loss of generality, we assume that the dipoles are polarized along the $x$ axis in the rotating frame. The effective quasi-2D dipolar interaction potential for this case has a similar form as Eq. (5) with $f_{\lambda}^{z}(\varrho)$ replaced by

$$
\begin{aligned}
f_{\lambda}^{x}(\varrho) & =\frac{\lambda^{3 / 2} e^{\lambda \varrho^{2} / 4}}{6} \frac{y^{2}-2 x^{2}}{\varrho^{2}}\left[\lambda \varrho^{2} K_{0}\left(\frac{\lambda \varrho^{2}}{4}\right)\right. \\
& \left.+\left(2-\lambda \varrho^{2}\right) K_{1}\left(\frac{\lambda \varrho^{2}}{4}\right)\right]+\frac{\sqrt{\pi \lambda}}{2 \varrho^{2}} U\left(\frac{3}{2}, 0, \frac{\lambda \varrho^{2}}{2}\right) .
\end{aligned}
$$

It can be easily shown that, unlike in the previous case, now the effective 2D dipolar interaction potential

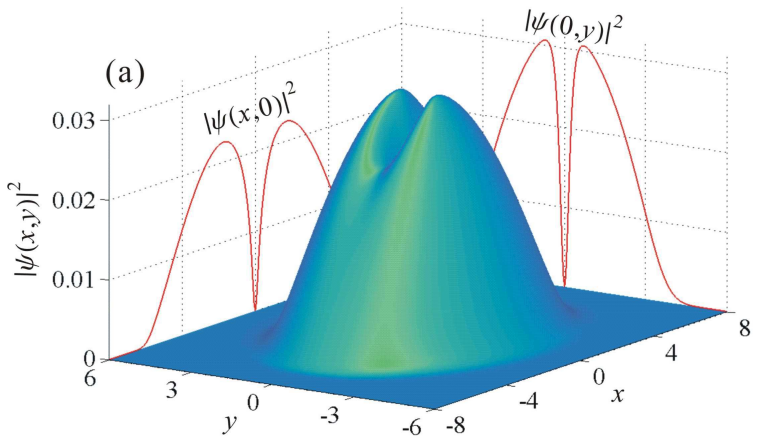

(b)

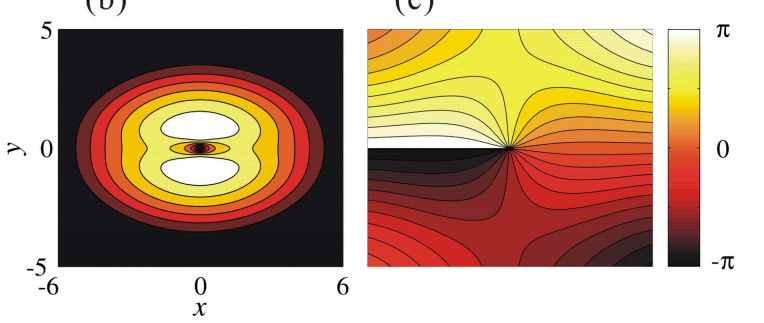

FIG. 4: (Color online). Structure of a single vortex state with dipoles polarized along the $x$ axis. The parameters are $\lambda=100, a=16 a_{B}$ and $\Omega=0.3 \omega_{\perp}$. (a) Density profile. The solid curves show the densities along the $x$ - and $y$-axis. (b) Density contour plot where the elliptical shaped vortex core can be clearly seen. Brighter color represents higher density. (c) Contour plot of the phase of the wave function.

is anisotropic: it is repulsive along $y$ axis and attractive along $x$ axis. Such a system is unstable for scattering lengths below a threshold value, which is about $15 a_{B}$ for the parameters used in our calculation.

Figure 4 shows the structure of a single vortex state for $a=16 a_{B}$. The whole atomic cloud in this case is elongated along the $x$ axis with a two-fold symmetry. This is due to the magnetostriction induced by the dipolar interaction, an effect that has been recently observed in experiment 15. One can also notice from Fig. 4(b) that the vortex core is also anisotropic: it has an elliptical shape with the major axis along $x$. This can be understood as follows: the vortex core size is determined by the healing length, and the attractive (repulsive) dipolar interaction along $x(y)$ direction weakens (enhances) the contact interaction, resulting in a larger (smaller) effective healing length, and hence core size, along $x(y)$.

Along any closed curves around the anisotropic vortex core, there is still a phase slip of $2 \pi$. However, the phase of the wave function no longer coincides with the azimuthal angle $\varphi$ [see Fig. [4(c)]. Generally, a vortex state with a two-fold symmetry can be represented as $\psi \sim \sum_{n} a_{n} \rho^{|2 n+1|} e^{i(2 n+1) \varphi}$. To a good approximation the vortex core structure shown in Fig. 4 can be modelled by the three most dominant terms with $n=0, \pm 1$, i.e., the wave function near the core takes the form

$$
\psi(\boldsymbol{\rho}) \sim \rho\left(e^{i \varphi}+\alpha e^{-i \varphi}\right)+\beta \rho^{3} e^{3 i \varphi},
$$




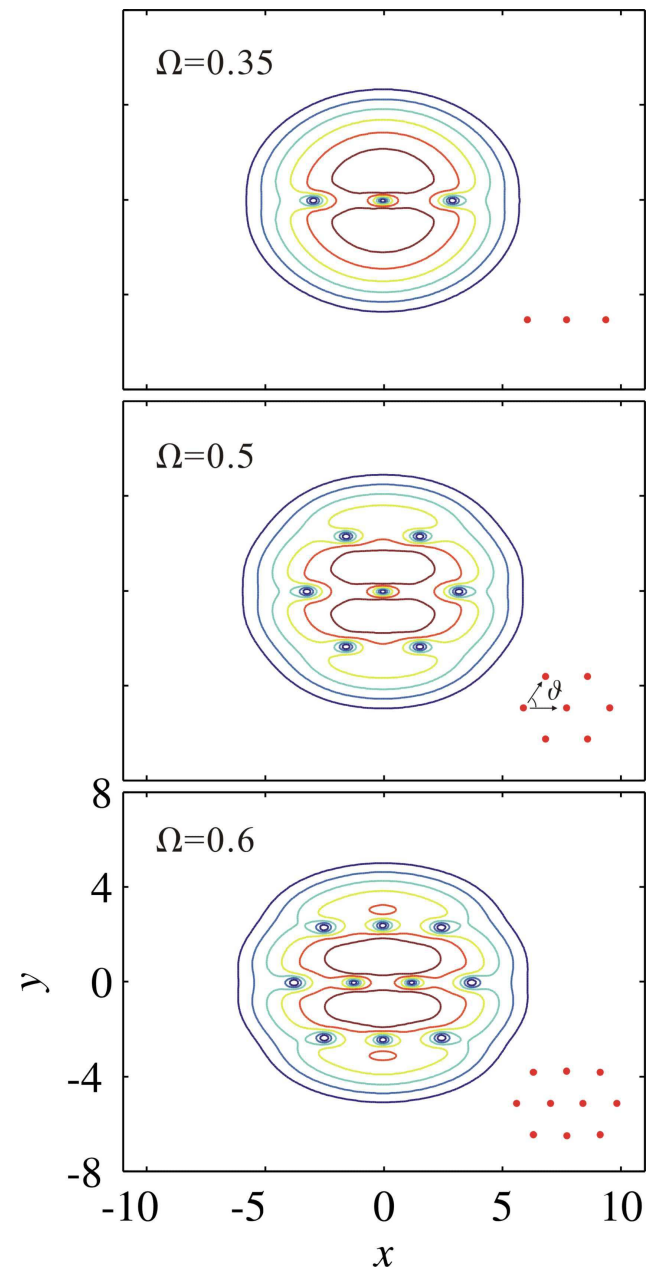

FIG. 5: (Color online). Density contour plots for $\lambda=100$, $a=18 a_{B}$ and various rotation frequencies. The dots at the lower right corner shows the position of vortices. For $\Omega=0.5$ and $0.6 \omega_{\perp}$, the angles $\vartheta$ are $55^{\circ}$ and $62^{\circ}$, respectively. with parameters $\alpha<0$ and $\beta>0$.

At higher rotation frequencies, vortex lattice will form with a structure strongly depending on the scattering length and the rotating frequency. The richness of the vortex lattice structures are illustrated in Fig. 5] for $a=18 a_{B}$ at three different rotation frequencies. For $\Omega=0.35 \omega_{\perp}$, three vortices appear, all located along the $x$ axis. Note that for the isotropic interaction as in the previous case or in a non-dipolar condensate, the threevortex state would have the three vortices situated on the corners of an equilateral triangle. At higher rotation frequency, the vortices form lattices with two-fold symmetry instead of the usual hexagonal symmetry. For $\Omega=0.5$ and $0.6 \omega_{\perp}$, the angles $\vartheta$ as defined in the middle plot of Fig. [5] are about $55^{\circ}$ and $62^{\circ}$, respectively. Numerically, it becomes increasingly difficult to find the lowest energy lattice structure when we further increase $\Omega$. We plan to study this problem in greater detail in a future work.

We remark that anisotropic vortices have been extensively studied in the context of high $T_{c}$ superconductors, where the anisotropy can arise from the crystal anisotropy [16] or from high angular momentum pairing interaction (e.g., in $d$-wave superconductors) 17]. In these anisotropic superconductors, the vortex lattice also deviates from the hexagonal geometry and can sometimes take a square shape [18].

In conclusion, we have investigated the properties of the vortex states of a quasi-2D rotating dipolar condensate. The vortex states possess many novel and unique features due to the presence of the dipolar interaction. We hope that our work will stimulate more experimental efforts on rotating dipolar quantum gases.

This work is supported by Rice University and the Oak Ridge Associated Universities.
[1] L. M. Pismen, Vortices in Nonlinear Fields: from Liquid Crystals to Superfluids, from Non-Equibrium Patterns to Cosmic Strings (Clarendon Press, Oxford, 1999).

[2] For a review of vortices in trapped condensates, see, for example, A. L. Fetter and A. A. Svidzinsky, J. Phys.: Condens. Matter 13, R135 (2001).

[3] M. W. Zwierlein et al., Nature (London) 435, 1047 (2005).

[4] A. Griesmaier et al., Phys. Rev. Lett. 94, 160401 (2004).

[5] For a review, see, for example, M. Baranov et al., Phys. Scr. T102, 74 (2002).

[6] J. Werner et al., Phys. Rev. Lett. 94, 183201 (2005).

[7] P. Pedri and L. Santos, cond-mat/0503019 Uwe R. Fischer, cond-mat/0506368

[8] M. Sadd, G. V. Chester, and L. Reatto, Phys. Rev. Lett. 79, 2490 (1997).

[9] N. G. Berloff and P. H. Roberts, J. Phys. A: Math. and Gen. 32, 5611 (1999).

[10] C. A. Sackett et al., Phys. Rev. Lett. 82, 876 (1999); J. L. Roberts et al., Phys. Rev. Lett. 86, 4211 (2001).
[11] H. Saito and M. Ueda, Phys. Rev. A 69, 013604 (2004).

[12] N. R. Cooper, E. H. Rezayi, and S. H. Simon, cond-mat/0505759

[13] J. Zhang and Hui Zhai, cond-mat/0506118

[14] If the dipoles are not co-rotating with the condensate, the dipolar interaction potential will have an explicit time dependence in the rotating frame. Such a system may not support a stationary state.

[15] J. Stuhler et al., cond-mat/0508228

[16] S. J. Bending and M. J. W. Dodgson, J. Phys.: Condens. Matter 17, R955 (2005).

[17] A. J. Berlinsky et al., Phys. Rev. Lett. 75, 2200 (1995); J.-H. Xu, Y. Ren and C.-S. Ting, Phys. Rev. B 53, R2991 (1996); M. Ichioka et al., Phys. Rev. B 53, 15316 (1996); M. Ichioka, A. Hasegawa, and K. Machida, Phys. Rev. B 59, 8902 (1999).

[18] I. Maggio-Aprile et al., Phys. Rev. Lett. 75, 2754 (1995); R. Gilardi et al., Phys. Rev. Lett. 88, 217003 (2002). 